\title{
Participation of Morphofunctional Zones in Aging Processes
}

\author{
T. M. Yavisheva, S. D. Shcherbakov \\ Blokhin Cancer Research Center, Russian Academy of Medical Sciences, Kashirskoe sh. 24, Moscow, Russia \\ Email: Javisheva@rambler.ru
}

Received 29 January 2014; revised 3 April 2014; accepted 22 April 2014

Copyright $@ 2014$ by authors and Scientific Research Publishing Inc.

This work is licensed under the Creative Commons Attribution International License (CC BY). http://creativecommons.org/licenses/by/4.0/

(c) (i) Open Access

\begin{abstract}
There are morphofunctional zones in organism tissues, where proliferation and differentiation processes occur. Daughter cells are differentiated in the electric field excited by 12 mother and daughter cell pairs, which turned out at cambial cell division. With aging, the cambial cell number is reduced to 7, which is close to threshold level ( 6 cells), at which the differentiation of daughter cells is absent. The depression of cambial cell number with aging is connected with the work of another morphofunctional zone- the hypothalamus, which is the major center of vegetative regulation and initially has very high RhoA activity, which has been established in embryogenesis. Estrogens, influencing over the hypothalamus and activating Src kinase in its nuclei, reduce level of RhoA activity, including SCN, responsible for many biorhythms of an organism. As a result, the hyperestrogenemia and therefore a connective tissue at first occur. Then there happens a hypoestrogenemia that leads to sharp falling of proliferative activity of cells, causing the depression of cambial cell number and possibility of a malignant tumor development. Along with this, there are the deep lesions of hormone regulation, leading to some lethal diseases. Thus, the RhoA increasing in hypothalamus and especially in SCN circadian rhythm can counteract the Src kinase intensifying and prevent the processes connected with this.
\end{abstract}

\section{Keywords}

Morphofunctional Zones, Hypothalamus, RhoA Protein, Src Kinase, Aging

\section{Structure and Function of Skin Morphofunctional Zones}

In our previous research, the spatial organization of different tissues had been revealed [1]. The identical principle of work occurs in various tissue structural units. Having analyzed the spatial organization of various tissues in age aspect, we have revealed some mechanisms, which participate in aging process. 
There are the morphofunctional zones in different epithelia, where proliferation and differentiation processes take place [1]. Zone consists of two subunits acting in turn. Each subunit includes 12 cambial cells, at which division are formed mother and daughter cells. Primarily, the cambial cells of one subunit undergo division and form 12 pairs of mother and daughter cells, and then of the other subunit also form 12 pairs. Differentiation of daughter cells can be passed in an electric field excited by 12 mother and daughter cell pairs, which are disposed in the same subunit. Each subunit undergoing proliferation does not have 12 cells entering mitosis together. At first, 6 cells of one subunit divide and only then do the other, i.e. up to half of all cells divide simultaneously. Thus, daughter cells from the first six pairs of mother and daughter cells are not stretched. Only because the amount of these pairs rises up to 12, due to the division of the other six cambial cells of the same subunit, the daughter cells lengthen along the basal membrane. Therefore 6 pairs of these cells form a field with a limit value, when daughter cell differentiation does not happen. When the amount of daughter cells in both subunits reaches a total of 24 items, 12 of them, formed earlier, and 12 others are then gradually transformed into reserve cells (30\%), providing physiological regeneration in the morphofunctional zone.

In the functioning of morphofunctional zone it may be distinguished by two key proteins: tyrosine kinase Src and RhoA, which participate in various paths of transduction and provide also the development of cell microfilaments [1]. These proteins define a direction of a long axis of cambial cell which unlike the other cells is normal with the basal membrane, instead of parallel with it. It happens at the expense of a strong RhoA expression and weak Src kinase in the cambial cell, which reduces the formation of stress fibers and microtubules and decreases the spreading of this cell along the basal membrane. As a result, one of received at its division cell remains attached to the basal membrane (mother), and another (daughter) cell settles down over it. Thus, the mother cell body, adjacent to the basal membrane remains spasm, and lateral edges are tightened up, i.e. the cell gets the canoe-like form. The large role is played thus by growth factors, as a transforming growth factor $\beta$ type, accumulating in a basal membrane and possessing very strong RhoA activity and spastic action. It protects a mother cell from a differentiation. Therefore the mother cell nucleus is not stretched, and the density of the chromatin having a negative charge, sharply increases in it in comparison with the daughter cell. It leads to redistribution of superficial charges between the pair of these cells and causes some electric field formation [1]. But it is not enough the force of one pair field for a distention and a differentiation of daughter cell. The differentiation of the latter happens only in the field of 12 pairs which have turned out at division of 12 cambial cells, which compound one subunit of morphofunctional zone. Formation of two subunits in the zone takes place in embryogenesis and is caused by certain relation between Src and RhoA in cells. It is known that blastomere which receives a part of egg cytoplasm, including sperm tail, enters the second division faster than a sister blastomere [2]. It happens because the sperm tail consists of microtubules in which formation of Src kinase participates, providing a cytokinesis. Therefore descendants of these cells always enter division faster than the others, which lead to separation of zone into two subunits.

\section{Two Key Proteins of Morphofunctional Zone: Src and Rhoa Define the Cell Differentiation and Proliferation}

There is a moderately activated Src kinase SH2 domain in daughter cells at their distention, whichis necessary for development of microfilaments. In chromosomes due to the certain nucleus periphery-binding sites, certain fields nearby telomeres are unwound, defining an epidermal cell differentiation [1]. Then, unlike charged mother and daughter cells conjoin forming a circular structure, which disbanding leads to the daughter cell transformation into other forms, i.e. differentiation. However epidermal daughter cells cannot be stretched in electric field without their preliminary cortex relaxation. This process is carried out due to dermal daughter cells. It emerged that derma has morphofunctional zones identical with the epidermal zones [1]. On the other side of the basal membrane, the derma papillary layer cambial cells function simultaneously with epidermal cells, synchronously enter mitosis and pass identical transformation stages. Thus, dermal mother and daughter cells turn out. In the latter under the influence of dermal microenvironment there is the Src kinase SH3 domain, actively expressed. There is a sharp decreasing of RhoA activity at a high expression of Src, because the latter phosphorylates p190 RhoGAP protein, which inactivates RhoA. Thus, formation of active formins drops and Rho kinase decreases, that leads to cortex relaxation and to depression of stress fibers formation in dermal daughter cells. Therefore the latter stretch sharply along voltage lines, that leads to strong activization of Src kinase SH2 domain, responsible for microtubules development. Thus, the chromosome loops unwinding is carried out in centromere-close man- 
ner, defining mesodermal differentiation of dermal daughter cells. The latter develop the growth factors strongly activating Src kinase SH3 domain. But there is only moderate stimulation of Src kinase SH3 domain in epidermal daughter cells under this influence, because the latter are constricted due to their microenvironment. It leads to some cortex relaxation of epidermal cells and possibility of their differentiation in electric field as Src kinase reduces RhoA activity. However, in epidermal daughter cells RhoA expression remains a little above Src kinase. In turn, epidermal daughter cells due to their growth factors, enhance RhoA activity in dermal daughter cells, promoting their proliferation. Participation of dermal component in the work of epidermal zone is caused by early embryonic development. So, gastrulation processes begin only at action of active Src kinase, expressed on the border of animal and vegetative region, on the egg animal region, where RhoA is strongly activated [3].

Growth factors of epidermal and dermal daughter cells in epidermal morphofunctional zone exert unlike effects not only to each other, but to cambial cells also. When there is a sharp prevalence of epidermal factors in the zone, which very strong activate RhoA, the cambial cells will be constricted and not active. When there is a rising of dermal cells growth factors, which stimulate Src kinase and reduce RhoA, then the proliferative activity of cambial cells will be increased. The cambial and daughter cells proliferate only under the condition, when there are considerably induced Src kinase and RhoA in them, and the latter more than moderate prevails over the former. It is connected with the fact, that Src kinase to some extent inactivates RhoA. Therefore more than moderate prevalence of RhoA over Src kinase leads to equal activation of these proteins. It will cause the production of many formins, necessary for the formation of actomyosin ring, and also microtubules of the division spindle, -which will provide a mitosis. Proliferation of cells does not happen under the other conditions. When RhoA strongly prevails over Src kinase, the cells spasm will be intensified. The influence of RhoA strengthens in epidermal morphofunctional zone with aging, which leads to cells spasm. It happens due to the reduction of the cambial cells number with aging and electric field weakening, which leads to aggravation of the daughter cells distention and depression of Src kinase SH2 domain in them, responsible for a differentiation. Over 75 years, the cambial cells number in one zone subunit is reduced to 7 , that is close to threshold level (6 cells), at which differentiation of daughter cells is not present or is very weak [4]. Hence, cambial cells number lowering down to 6 in one subunit of zone under the influence of unfavorable factors can lead to malignant tumor formation.

The aforesaid work of morphofunctional zone is schematic, because its functioning is influenced by many other factors, including the hormones, which reduce or intensify the expression of Src kinase and RhoA in it.

\section{Morphofunctional Zones of Other Tissues. A Hypothalamus Is a Main Morphofunctional Zone of the Organism}

Functional zones besides a skin exist in other tissues: a mammary gland, reproductive organs. In human testis, also, as in epidermis, only 6 various associations of epithelial cells being in one phase of a mitotic cycle and functioning simultaneously in the same time interval are revealed. An ovary also has morphofunctional zones according to its functional features. At multiparous animals in both ovaries about 20 follicles mature simultaneously, that corresponds to cambial cells number in morphofunctional zone. Besides, during the monthly secretion of a pituitary follicle-stimulating hormone (FSH), only a small part of antral follicles in ovaries start to grow. Apparently, in each ovary or a testis only one subunit cells are presented. Under the influence of hormonal factors at first one subunit cells in one ovary or a testis begin to work, and then - cells of the other subunit already in the second ovary or a testis.

The hypothalamus with its hypophysiotropic region also is the morphofunctional zone and the important vegetative center of a brain. Here, as well as in epidermal morphofunctional zone, RhoA expression prevails over Src kinase. Therefore, the majority of the factors, developed by a hypothalamus, stimulate RhoA much stronger, than Src kinase. It is caused by brain development from an ectodermal tissue with high RhoA expression in embryogenesis. During the formation of the neural plate due to the influence of a subject chorda with strong Src kinase expression, there is a fast and transitional Src activization in neural tissue. Thus there is an activation of receptors of epidermal growth factor (EGF). We reported earlier, that these receptors possess low affinity to Src in comparison with receptors of fibroblasts growth factor (FGF) [1]. Hence, moderate expression of Src and p190RhoGAP protein, inactivating RhoA, considerably will enhance RhoA in these cells in embryogenesis. And, the more close to neural plate edges, the strongly will be the RhoA stimulation, because chorda influence is reduced here. Therefore, one brain factors will stimulate RhoA stronger, and others-weaker, but RhoA expres- 
sion will prevail over Src in all these factors. Hypothalamic and epidermal morphofunctional zones function principles are identical. Signals come to a hypothalamus from surrounding neocortex, subcortical structures, and also from organs-targets (reproductive organs, thyroid, adrenal cortex). If coming signals very strong intensify RhoA expression in hypothalamus nuclei, the latter will not develop the releasing factors. Because high RhoA expression in relation to Src kinase enhances the cells' and intracellular structures' spasm, which leads to depression of exocytosis and transcription of the corresponding proteins in hypothalamus nuclei. If coming signals reduce RhoA expression in relation to Src kinase and therefore remove the block, the peptidergic neurons will produce regulation factors. It is shown above, that all hypothalamic factors differ from each other by a degree of RhoA expression. Therefore the coming signal can be activating for one releasing factors, i.e. when RhoA/Src of this signal coincides with that in factors and inhibiting for others, in which this correlation is lower. Further the information is passed to the pituitary, and then to the corresponding glands, which start to produce hormones with the certain prevalence of RhoA over Src kinase. The hypothalamus will produce regulation factor for the certain hormone until general RhoA expression of the secreted hormone in organ-target is lower, than in hypothalamus (a positive feedback). But when RhoA is higher, then the neurons' function will be inhibited, because of a spasm, which is developed due to the RhoA action of all secreted hormone on them (a negative feedback). The same principle of regulation occurs not only at a level of a long loop of the feedback (organs-targets-a hypothalamus), but also of a short (anterior pituitary—a hypothalamus) and ultrashort (releasing factors-neurocytes) loops.

However, this scheme of regulation is more complicated, because the function of many peptidergic neurons is corrected by the neurons of supra-chiasmatic nucleus (SCN), which are responsible for various biological rhythms in organism. SCN neurons project directly to the medial preoptic, paraventricular and dorsomedial nuclei and the subparaventricular region of the hypothalamus. Exciting impulse comes on SCN from photoreceptors and ganglion retina cells, which contain the photosensitive pigment melanopsin. Thus, not only electrical impulse, but also MAP kinase (mitogen-activated protein kinase)-c-Fos pathway is generated [5] [6], evidencing about Src kinase activation in neurons in ventrolateral SCN. Neurons in other dorsomedial SCN have own, independent of light, approximately the 24-hour rhythm with the maximum activity in the afternoon and minimum - at night. Neurons of these two parts have close contacts, which provide Src kinase activation at light in the dorsomedial part too. But Src kinase reduces RhoA, because it activates p190RhoGAP, promoting inactivation of RhoA. Therefore, the RhoA expression and the production of transmitters with high RhoA activity in SNC neurons at light will be lowered, that will cause the inhibition of noradrenergic transmission. It in turn will lead to depression of noradrenaline release in the epiphysis and to decreasing of the enzymes activity, necessary for melatonin synthesis. In absence of light the neurons in dorsomedial SCN function in the usual 24-hour rhythm, but melatonin corrects their work. Really, the epiphysis, as well as other brain structures influences over hypothalamic morphofunctional zone. In the epiphysis there are various regulatory factors with high and very high RhoA activity, in particular, noradrenaline, serotonin, melatonin, and etc. Especially, many melatonin receptors are revealed in SCN. Possessing very high RhoA activity, melatonin at night promotes a spasm of SCN neurons, which reduces their metabolism and therefore enlarges duration of their own cycle. Besides, the exocytosis and releasing of the transmitters, which pass the signals to the other hypothalamic nuclei, dependent on SCN, get worse. Therefore the high maintenance of melatonin in prepuberty depresses the secretion of many hypothalamic releasing factors.

\section{Participation of the Hypothalamus and Key Proteins: Src and Rhoa in the Processes of Aging}

In puberty the maintenance of melatonin decreases 40 times. It leads to weakening of SCN and other hypothalamic neurons spasm. The transcription of neurotransmitters and their exocytosis are increased thus, that enlarges the secretion of hypothalamic, releasing factors. Epiphysis melatonin depression and, hence, decreasing of hypothalamic RhoA activity is possible for the following reason. In the adrenal cortex in prepuberty there is a small synthesis of estrogens, because adrenocorticotropic hormone (ACTH) is not completely blocked by melatonin. Besides, synthesis of estrogens takes place in the hypothalamus and other brain departments [7]. It is known, that estrogens are in turn synthesized from their precursor-cholesterol. Its accumulation in brain cells, adipose tissue, gonads, adrenal cortex is the basis for the further synthesis of steroid hormones. At children of 5 - 7 years old the level of cholesterol and cholic acids is lower, than at the adult, which is caused by ontogenetic 
mechanism of fermental systems maturation [8]. There are receptors to estrogens in hypothalamus and epiphysis cells. But estrogens strongly activate Src kinase by transactivation of EGF receptors or activization of G-proteincoupled receptors: subunits Gai and Gas, which directly co-operate with catalytic domain of Src kinase and activate it [9]. It reduces the level of RhoA in hypothalamus and epiphysis cells and leads to the depression of melatonin synthesis and to removal of the block from all releasing factors of the hypothalamus. Hence, increasing of estrogens synthesis, which in turn occurs in organism during the process of ontogenetic cholesterol acquiring, leads to depression of RhoA activity in the hypothalamus, especially in SCN. It causes activization of hormonal system and puberty. Interestingly, in puberty along with cholesterol there is the accumulation of lipochromes in brain, adipose tissue, adrenals, gonads [10]. Cholesterol and lipochromes are the integral component of membranes. Lipochromes belong to the class of lipids and consequently start to be acquired simultaneously with cholesterol as the cholic acids necessary for their emulsification and formation of micelles are formed of cholesterol. Lipochromes and cholesterol are derivatives of the "active" isoprene containing isoprene units. Lipochromes, very unstable substance and at stabilization can be removed from the organism. If they are not stabilized, they can disintegrate with formation of isoprene units. But the latter serve to synthesis of cholesterol and steroids. Interestingly, some part of lipochromes of ovaries is associated with proteins, whereas the lipochromes of testis are not associated, which provides their fast disintegration and participation in synthesis. Obviously, lipochromes participate not only in protection of cells from peroxidative oxidation, but also in intensifying of cholesterol and estrogens synthesis.

Thus, in puberty owing to depression of strong braking melatonin action, the neurotransmitters with lower in comparison with melatonin RhoA expression are activated. Therefore SCN passes much more signals with certain RhoA expression to the peptidergic neurons, which enhance production of various releasing factors, including a gonadotropin-releasing hormone (GnRH). The latter activates a luteinizing hormone (LH) and FSH. LH possesses high enough RhoA activity and stimulates synthesis of androgens in granulosa and theca cells, and FSH enhances androgen aromatization with estrogen formation, i.e. activates Src kinase. The latter leads to intensive proliferation of granulosa cells as participates in cytokinesis. Under the influence of FSH these cells produce the increasing quantity of estradiol, which enhances Src kinase expression in the hypothalamic center, synthesizing GnRH. Thus, the portion of luteinizing hormone-releasing hormone (LHRH) will be decreased in GnRH, because Src kinase reduces RhoA. It will cause the development of a follicular phase of a cycle in which Src kinase expression and accordingly a pituitary FSH prevail. The lowered ratio of LHRH cannot block high enough RhoA-signal which has come from SCN. Therefore the secretion of LHRH will occur until its general RhoA-expression does not exceed a signal from SCN neurons. If it exceeds, it will inhibit the SCN neurons in ultrashort loop. Thus a quantity of LHRH and its RhoA activity will gradually raise, which finally will lead to SCN block. Thus the maximum output of LHRH and accordingly of the pituitary LH will be provided, that will cause an ovulation. Hence, in an ovulation there is Src kinase increasing and maximum RhoA development. The basic condition, necessary for the proliferation of cell, including oocyte is thus observed: strong Src kinase and RhoA expression, with more than moderate prevalence of RhoA. It in turn leads to development of actomyosin ring and the first polar body in oocyte [3]. Along with this, appreciable Rho expression of LHRH will start to prevent from Src kinase intensifying in the hypothalamic sexual center. It in turn will cause depression of pituitary FSH production. The fractional composition of estrogens, taken at the end of a follicular phase, argues about it: estriol level is sharply enlarged along with estradiol in urine. The estriol, is known to be the final product of estradiol and estron metabolism and possesses low affinity to receptors of estrogen-competent cells. Its augmentation at the end of a follicular phase, before an ovulation argues about the beginning of FSH inhibition. Men do not have cyclic LH output as estrogens constantly and are in regular form produced in testis and brain. Therefore estrogens cannot sharply lower the level of LHRH, and the development of the latter will occur in a usual regime, i.e. tonically.

It is mentioned previously, that LH and FSH activization is carried out by the same GnRH. Really, the exciting signal with high enough RhoA expression comes from SCN to GnRH and activates the LHRH with strong RhoA activity. It leads to exocytosis retardation and decreasing of LHRH output from neurocytes at the expense of cellular structures spasm. As a result, its pulse frequency becomes rarely, which leads to only moderate intensifying of its concentration and receptors in the corresponding pituitary cells. But as LHRH acts on pituitary cells through EGF receptors, therefore in pituitary cells will be strongly expressed RhoA and moderately Src kinase, that will lead to LH activization. Intensifying of estradiol influence during the follicular phase and augmentation at the expense of this the Src kinase expression in the neurocytes, producing GnRH, leads to im- 
provement of exocytosis and a metabolism of neurocytes. It leads to the increasing of pulse frequency of GnRH and considerable augmentation of its concentration and receptors number on the corresponding pituitary cells. It is known, that under such conditions there is a transactivation of EGF receptors, which leads to strong Src kinase activization, as under FGF influence. Thus in pituitary cells will activate the FSH synthesis, in which Src kinase activity prevails over RhoA.

Selection of the unique dominant follicle, designed to an ovulation, can be explained by the following. The work of the ovaries oocytes, as mentioned previously, takes place in morphofunctional zones, therefore in each cycle both ovaries function. At first in one, then in other ovary act in turn 12 primordial follicles, which start to grow, maturating into preantral follicles. For this stage the activization of estrogen production, which is developed in granulosa layer, is characteristic. A dominant follicle will be the follicle, which has the greatest estrogen activity, expressing Src kinase in its cells. Apparently, it is one of the first synchronized 6 follicles which have entered a cycle. Really, activization of proliferation in granulosa cells and the formation of the first polar body in the oocyte is possible only at high enough Src kinase and RhoA level, and the abundant expression of the latter occurs at maximum ovulatory LH output. In case of average or low Src expression in granulosa cells and in oocyte instead of formins, which participate in actomyosin ring formation, short actin cords, which force is insufficient for a cytokinesis, will be formed. It will lead to cortical layer spasm of such granulosa cells and oocytes, that will cause their transcription depression and atresia. Interstitial cells of theca interna grow instead of the lost cells, which at the LH influence develop the androgens, turning into estrogens under FSH influence. Hence, with aging during follicles destruction, it is gradually enlarged the ovaries estrogen activity. But estrogens activate Src kinase and influence over SCN and all hypothalamic zone, and also over the epiphysis, and reduce RhoA expression in hypothalamic nuclei. Owing to the depression of inhibiting RhoA action in the hypothalamus, FSH and Src kinase stimulation in the pituitary will be strongly enlarged with aging,which will reduce LH activity to some degree. Therefore,the FSH level will exceed LH, that will lead to increasing of anovulatory cycle number. Later the LH level also begins to increase in the pituitary. Really, the transmitters number, activating LHRH, decreases with aging, therefore it will be developed less. It is the result of RhoA depression, that leads to the falling of transmitters formation and intensifying of the exocytosis, reducing their deposition. LHRH will inhibit its own production, if its general RhoA expression is higher, than in a signal coming from SCN. Therefore hypothalamic neurocytes will continue to synthesize LHRH, which enlarges LH level. However LH increasing is late in relation to FSH and is not higher, that leads later to impossibility of contractile ring formation in oocyte and to firm absence of the ovulation, that corresponds to a menopause.

Thus, in ovaries during a hyperestrogenemia period the Src kinase expression will prevail over RhoA. We previously reported, that such prevalence leads to a distention of cells nuclei more close to centromeres and to formation of fibroblastlike cells. Hence, in ovaries sclerosis process at the expense of the development of connective tissue will be intensified. It will lead to attenuation of ovaries hormone function, that in turn leads to a hypoestrogenemia in a postmenopause. Along with ovaries, there is the sclerosis process in brain thanks to Src kinase augmentation in relation to RhoA, which leads to reduction of neurons number and parenchymatous tissue in the epiphysis. It in turn causes still larger depression of neurotransmitters production by the epiphysis, which attenuates the brain RhoA activity, especially of hypothalamic zone. Thus, RhoA expression in SCN circadian cycle sharply weakens. Hence, rising of the threshold of the hypothalamus sensitivity with aging means the gradual RhoA depression in the hypothalamus and especially in SCN.

Decreasing of the hypothalamus and brain RhoA activity with aging also causes the imbalance of the expression of other releasing factors of a hypothalamus and pituitary hormones. It is mentioned previously, that in the young persons melatonin and others neurotransmitters, possessing very high RhoA activity, reduce a metabolism of SCN neurons, regulating biological rhythms, therefore duration of their own cycle is enlarged. With aging due to the neurotransmitters number falling, there is the decreasing of their retarding influence over SCN, therefore the circadian cycle of the latter is shortened. Hence, at aging many releasing factors of a hypothalamus will be developed at earlier o'clock. For example, the adrenocorticotropic hormone (ACTH) instead of 6 - 8 a.m. in junior age is developed at $3 \mathrm{a}$.m. in elderly persons, that leads to relative augmentation of this hormone quantity. Conditions for atherosclerosis development are thus formed. At men the atherosclerosis lesions of cardiovascular system happen much earlier, than at women. Because testosteron, that is produced by testis, turns in brain to estrogens, which in larger quantity and constantly, unlike women, influence over the hypothalamus and consequently, to the greater degree, reduce its RhoA activity.

At appreciable depression of transmitters with high RhoA activity, there is the absolute ACTH ascending. Re- 
ally, the small transmitters number with high enough RhoA expression, passing the signal from SCN, can activate not much hypothalamus corticotpopin-releasing hormone. The latter will be developed until the general RhoA activity of this releasing hormone does not prevail the RhoA activity of transmitters. Otherwise, it will inhibit own development in the ultrashort loop (negative feedback). Accordingly to this, there will be the pituitary ACTH increasing, because its much more production is necessary for inhibition in a short loop. The information from the pituitary is passed to adrenals, which start to develop more glucocorticoids by the same principle. The firm rising of ACTH quantity leads to metabolic stress, diabetes development, an atherosclerosis and finally to lethal exit from a cardiovascular pathology.

In the hyperestrogenemia period there is a superfluous influence of estrogens not only over organs-targets, but also other peripheric organs, because estrogens are hormones of a wide biological action. It leads to intensifying of Src kinase expression in tissues of various organs. As a result there is the rearrangement of the epithelial cells cytoskeleton, and their distention is closer to centromeres, that leads to their gradual transformation into connective tissue cells. Therefore, there are a connective tissue growth and depression of parenchymatous organs functions with aging. Then, in a hypoestrogenemia period, the Src expression drops, that leads to attenuation of mitotic activity of peripheric organs cells, as Src kinase participates in cytokinesis mechanisms. Not accidentally, in our research the first decreasing of cambial cells number is revealed just in 60 years during a hypoestrogenemia [4]. Scr kinase depression in this period leads to RhoA rising in cells, that enhances their constriction. The cells rigidity and weakening of their distention at the expense of the decreasing of cambial cells number leads to risk of the malignant tumor occurrence with aging, because at cells number lowering down to 6 there is no normal differentiation of daughter cells.

\section{Conclusion}

Thus, the carried out analysis has revealed that puberty starts processes of aging and organism death from a cardiovascular pathology, malignant tumors and other diseases at the expense of decreasing of hypothalamus RhoA activity under the influence of increasing estrogens quantity, intensifying Src kinase expression and RhoA decreasing in hypothalamus, especially in SCN. It leads to the work disturbance of the latter and to decreasing of neurotransmitters number with high RhoA expression, which results further in gradual fading of cell division in peripheric organs; to work disturbance of morphofunctional zones, to development of the connective tissue and to some lethal diseases. Hence, RhoA rising in the hypothalamus and especially in SCN circadian rhythm can counteract the Src kinase intensifying and prevent the processes connected with this.

\section{References}

[1] Yavisheva, T. and Shcherbakov, S. (2011) Characteristic Features of Proliferation and Differentiation of Cambial and Daughter Cells in Morphofunctional Zones in Normal Epithelium and Cancer in Age Aspect. Advances in Gerontology, 1, 50-56. http://dx.doi.org/10.1134/S2079057011010140

[2] Diban, A.P. (1988) Early Development of Mammals. Nauka, Leningrad.

[3] Yavisheva, T. and Shcherbakov, S. (2012) On the General Mechanisms of Both Somatic Cambial Cells and Germ Cells Proliferation, and the Concept of Cambial Cells. International Journal of Biomedicine, 4, 242-244.

[4] Yavisheva, T. and Shcherbakov, S. (2009) Morphofunctional Cambial Cells Changes and Their Derivates in Human Skin with Aging. Bulletin of Experimental Biology and Medicine, 9, 326-329.

[5] Bernard, S., Gonze, D., Cajavec, B., Herzel, H. and Kramer, A. (2007) Synchronization-Induced Rhythmicity of Circadian Oscillators in the Suprachiasmatic Nucleus. PLos Computational Biology, 4, 68. http://dx.doi.org/10.1371/journal.pcbi.0030068

[6] Toh, K.L. (2008) Basic Science Review on Circadian Rhythm Biology and Circadian Sleep Disorders. Annals of Academy of Medicine Singapore, 37, 662-668.

[7] Teppermam, J. and Tepperman, H. (1989) Metabolic and Endocrine Physiology. Mir, Moscow.

[8] Mazurin, A. (1984) Diseases of Digestion Organs in Children. Meditsina, Moscow.

[9] Razandi, M., Pedram, A., Park, S.T. and Levin, E.R. (2003) Proximal Events in Signaling by Plasma Membrane Estrogen Receptors. The Journal of Biological Chemistry, 4, 2701-2712. http://dx.doi.org/10.1074/jbc.M205692200

[10] Anisimov, V. and Vinogradova, I. (2008) The Aging of Woman Reproductive System. Sistema, S-Peter. 